The International Journal of Multimedia \& Its Applications (IJMA) Vol.6, No.3, June 2014

\title{
PERFORMANCE ANALYSIS OF IMAGE DENOISING WITH WAVELET THRESHOLDING METHODS FOR DIFFERENT LEVELS OF DECOMPOSITION
}

\author{
Anutam $^{1}$ and Rajni ${ }^{2}$ \\ ${ }^{1}$ Research Scholar SBSSTC, Ferozepur, Punjab \\ ${ }^{2}$ Associate Professor SBSSTC, Ferozepur, Punjab
}

\begin{abstract}
Image Denoising is an important part of diverse image processing and computer vision problems. The important property of a good image denoising model is that it should completely remove noise as far as possible as well as preserve edges. One of the most powerful and perspective approaches in this area is image denoising using discrete wavelet transform (DWT). In this paper, comparison of various Wavelets at different decomposition levels has been done. As number of levels increased, Peak Signal to Noise Ratio (PSNR) of image gets decreased whereas Mean Absolute Error (MAE) and Mean Square Error (MSE) get increased. A comparison of filters and various wavelet based methods has also been carried out to denoise the image. The simulation results reveal that wavelet based Bayes shrinkage method outperforms other methods.
\end{abstract}

\section{KEYWORDS}

Denoising, Filters, Wavelet Transform, Wavelet Thresholding

\section{INTRODUCTION}

Applications of digital world such as Digital cameras, Magnetic Resonance Imaging (MRI), Satellite Television and Geographical Information System (GIS) has increased the use of digital images. Generally, data sets collected by image sensors are contaminated by noise. Imperfect instruments, problems with data acquisition process, and interfering natural phenomena can all corrupt the data of interest [1]. Various types of noise present in image are Gaussian noise, Salt \& Pepper noise and Speckle noise. Image denoising techniques are used to prevent these types of noises while retaining the important signal features [2]. Spatial filters like mean and median filter are used to remove the noise from image. But the disadvantage of spatial filters is that these filters not only smooth the data to reduce noise but also blur edges in image. Therefore, Wavelet Transform is used to preserve the edges of image [3]. It is a powerful tool of signal or image processing for its multi-resolution possibilities.

This paper is organized as follows: Section 2 presents types of noise. Section 3 presents Filtering techniques. Section 4 discusses Wavelet based denoising techniques and various thresholding methods. Finally, simulated results and conclusions are presented in Section 5 and 6 respectively. 
The International Journal of Multimedia \& Its Applications (IJMA) Vol.6, No.3, June 2014

\section{2 .TYPES OF NOISE}

Various types of noise have their own characteristics and are inherent in images in different ways.

\subsection{Amplifier Noise (Gaussian Noise)}

The standard model of amplifier noise is additive, Gaussian, which is independent at each pixel and independent of the signal intensity. In color cameras, blue colour channels are more amplified than red or green channel, therefore, blue channel generates more noise [4].

\subsection{Impulsive Noise}

Impulsive noise is also called as salt-and- pepper noise or spike noise. This kind of noise is usually seen on images. It consists of white and black pixels. An image containing salt and pepper noise consists of two regions i.e. bright and dark regions. Bright regions consist of dark pixels whereas dark regions consist of bright pixels. Transmitted bit errors, analog-to-digital converter errors and dead pixels contain this type of noise [5].

\subsection{Speckle Noise}

Speckle noise is a multiplicative noise. It is a granular noise that commonly exists in and the active radar and synthetic aperture radar (SAR) images. Speckle noise increases the mean grey level of a local area. It is causing difficulties for image analysis in SAR images. It is mainly due to coherent processing of backscattered signals from multiple distributed targets [4].

\section{FILTERING TECHNIQUES}

The filters that are used for removing noise are Mean filter and Median filter.

\subsection{Mean Filter}

The advantage of using this filter is that it provides smoothness to an image by reducing the intensity variations between the adjacent pixels [6]. Mean filter is essentially an averaging filter. It applies mask over each pixel in signal. Therefore, to make a single pixel each of the components of pixel which falls under the mask are average filter. The main disadvantage of Mean filter is that it cannot preserve edges.

\subsection{Median Filter}

One type of non linear filter is Median filter. By firstly finding the median value and then replacing each entry in the window with the pixel's median value, median filtering is done [7]. Median is just the middle value after all the entries made in window are sorted numerically, if window has an odd number of entries. There is more than one median when window has an even number of entries. It is a robust filter. To provide smoothness in image processing and time series processing, median filters are used.

\section{WAVELET TRANSFORM}

Wavelet domain is advantageous because DWT make the signal energy concentrate in a small number of coefficients, hence, the DWT of a noisy image consists of number of coefficients having high Signal to Noise Ratio(SNR) while relatively large number of coefficients is having low SNR. After removing the coefficients with low SNR, the image is reconstructed using inverse DWT [3]. Time and frequency localization is simultaneously provided by Wavelet transform. Moreover, wavelet methods represent such signals much more efficiently than either the original domain or fourier transform [8]. 
The International Journal of Multimedia \& Its Applications (IJMA) Vol.6, No.3, June 2014

The DWT is same as hierarchical sub band system where the sub bands are logarithmically spaced in frequency and represent octave-band decomposition. Image is decomposed into four sub-bands and critically sampled by applying DWT as shown in Fig. 1(a). These sub bands are formed by separable applications of horizontal and vertical filters. Sub-bands with label LH1, HL1 and HH1 correspond to finest scale coefficient while sub-band LL1 represent coarse level coefficients [9] [3]. The LL1 sub band is further decomposed and critically sampled to find out the next coarse level of wavelet coefficients as shown in Fig. 1(b). It results in two level wavelet decomposition.

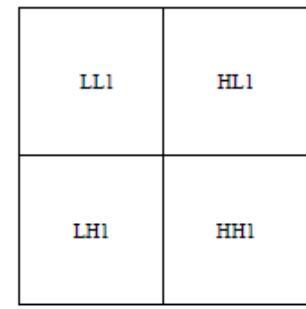

(a) One- Level

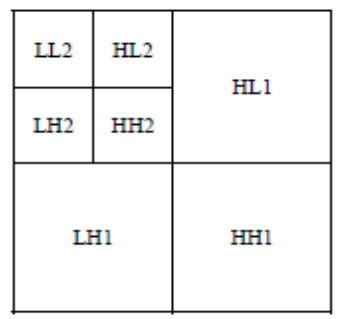

(b) Two- Level

Figure1. Image Decomposition by using DWT

\subsection{Wavelet Based Thresholding}

Wavelet thresholding is a signal estimation technique that exploits the capabilities of Wavelet transform for signal denoising. It removes noise by killing coefficients that are irrelevant relative to some threshold [9] .Several studies are there on thresholding the Wavelet coefficients. The process, commonly called Wavelet Shrinkage, consists of following main stages:

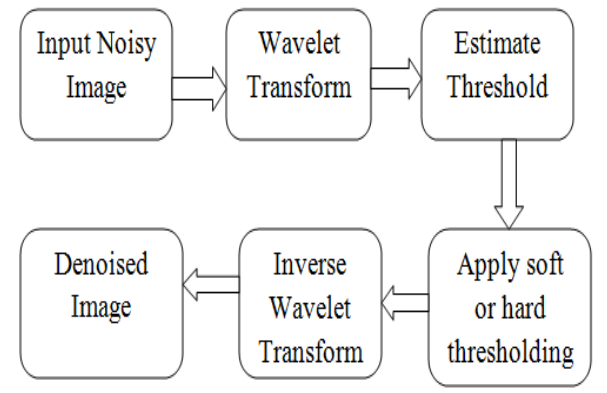

Figure2. Block diagram of Image denoising using Wavelet Transform

- Read the noisy image as input

- Perform DWT of noisy image and obtain Wavelet coefficients

- Estimate noise variance from noisy image

- Calculate threshold value using various threshold selection rules or shrinkage rules

- Apply soft or hard thresholding function to noisy coefficients

- Perform the inverse DWT to reconstruct the denoised image.

\subsubsection{Thresholding Method}

Hard and soft thresholding techniques are used for purpose of image denoising. Keep and kill rule which is not only instinctively appealing but also introduces artifacts in the recovered images is the basis of hard thresholding [10] whereas shrink and kill rule which shrinks the coefficients above the threshold in absolute value is the basis of soft thresholding [11]. As soft thresholding 
gives more visually pleasant image and reduces the abrupt sharp changes that occurs in hard thresholding, therefore soft thresholding is preferred over hard thresholding [12] [13].

The Hard Thresholding operator [14] is defined as,

$\mathrm{D}(\mathrm{U}, \lambda)=\mathrm{U}$ for all $|\mathrm{U}|>\lambda$

$$
=0 \text { otherwise }
$$

The Soft Thresholding operation the other hand is defined as,

$\mathrm{D}(\mathrm{U}, \lambda)=\operatorname{sgn}(\mathrm{U}) * \max (0, \mid \mathrm{UI}-\lambda)$

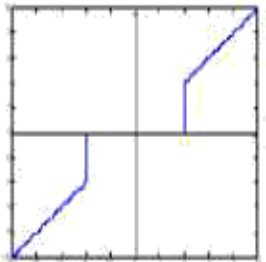

(a) Hard Thresholding

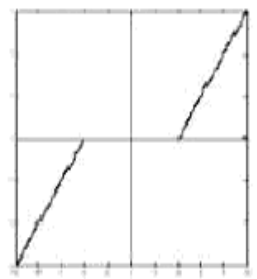

(b) Soft Thresholding [15]

Figure 3. Thresholding Methods

\subsubsection{Threshold Selection Rules}

In image denoising applications, PSNR needs to be maximized, hence optimal value should be selected [9]. Finding an optimal value for thresholding is not an easy task. If we select a smaller threshold then it will pass all the noisy coefficients and hence resultant images may still be noisy but larger threshold makes more number of coefficients to zero, which provides smoothness in image and image processing may cause blur and artifacts, and hence the resultant images may lose some signal values [16].

\subsubsection{Universal Threshold}

$$
\mathrm{T}=\sigma \sqrt{2 \log \mathrm{M}}
$$

where $\sigma^{2}$ being the noise variance and $\mathrm{M}$ is the number of pixels [17] .It is optimal threshold in asymptotic sense and minimizes the cost function of difference between the function. It is assumed that if number of samples is large, then the universal threshold may give better estimate for soft threshold [18].

\subsubsection{Visu Shrink}

Visu Shrink was introduced by Donoho [19]. It follows hard threshold rule. The drawback of this shrinkage is that neither speckle noise can be removed nor MSE can be minimized .It can only deal with additive noise [20]. Threshold T can be calculated using the formulae [21],

$$
\begin{aligned}
& T_{v}=\hat{\sigma} \sqrt{2 \log N} \\
& \hat{\sigma}^{2}=\left[\frac{\operatorname{median}\left(\left|X_{i j}\right|\right)}{0.675}\right]^{2}, X_{i j} \in H H 1
\end{aligned}
$$

Where $\sigma$ is calculated as mean of absolute difference (MAD) which is a robust estimator and $\mathrm{N}$ represents the size of original image. 
The International Journal of Multimedia \& Its Applications (IJMA) Vol.6, No.3, June 2014

\subsubsection{Bayes Shrink}

The Bayes Shrink method has been attracting attention recently as an algorithm for setting different thresholds for every sub band. Here sub-bands refer to frequency bands that are different from each other in level and direction [22]. Bayes Shrink uses soft thresholding. The purpose of this method is to estimate a threshold value that minimizes the Bayesian risk assuming Generalized Gaussian Distribution (GGD) prior [13]. Bayes threshold is defined as [23],

$$
t_{B}=\sigma^{2} / \sigma_{S}
$$

Where $\sigma^{2}$ is the noise variance and $\sigma_{s}$ is signal variance without noise.

From the definition of additive noise we have,

$$
w(x, y)=s(x, y)+n(x, y)
$$

Since the noise and the signal are independent of each other, it can be stated that,

$$
\sigma_{w}^{2}=\sigma_{s}^{2}+\sigma^{2}
$$

$\sigma_{w}^{2}$ can be computed as shown below:

$$
\sigma_{w}^{2}=\frac{1}{n^{2}} \sum_{x, y=1}^{n} w^{2}(\mathrm{x}, \mathrm{y})
$$

The variance of the signal, $\sigma_{s}^{2}$ is computed as

$$
\sigma_{s}=\sqrt{\max \left(\sigma_{w}^{2}-\sigma^{2}, 0\right)}
$$

\section{SIMULATION RESULTS}

Simulated results have been carried on Cameraman image by adding two types of noise such as Gaussian noise and Speckle noise. The level of noise variance has also been varied after selecting the type of noise. Denoising is done using two filters Mean filter and Median filter and three Wavelet based methods i.e. Universal threshold, Visu shrink and Bayes shrink. Results are shown through comparison among them. Comparison is being made on basis of some evaluated parameters. Also the comparison of wavelet thresholding methods at different decomposition level has been discussed. The parameters are Peak Signal to noise Ratio (PSNR), Mean Square Error (MSE) and Mean Absolute Error (MAE).

$$
\begin{gathered}
\text { PSNR }=10 \log _{10}\left(\frac{255^{2}}{M S E}\right) \mathrm{db} \\
\mathrm{MSE}=\frac{1}{M N} \sum_{i=1}^{M}(\mathrm{x}, \mathrm{y}) \sum_{j=1}^{N}(\mathrm{X}(\mathrm{i}, \mathrm{j})-P(i, j))^{2} \\
\mathrm{MAE}=\frac{1}{M N} \sum_{i=1}^{M}(\mathrm{x}, \mathrm{y}) \sum_{j=1}^{N}|\mathrm{X}(\mathrm{i}, \mathrm{j})-P(i, j)|
\end{gathered}
$$

Where, M-Width of Image, $\quad$ N-Height of Image P- Noisy Image , X-Original Image

Table 1 and Table 2 show the comparison of PSNR and MSE for cameraman image at various noise variancies. Figure 4 and Figure 5 shows that bayes shrinkage has better PSNR and low MSE than filtering methods and other wavelet based thresholding techniques. 
The International Journal of Multimedia \& Its Applications (IJMA) Vol.6, No.3, June 2014

Table1. Comparison of PSNR for Cameraman image corrupted with Gaussian and Speckle noise at different Noise variances using db1 (Daubechies Wavelet)

\begin{tabular}{|c|c|c|c|c|c|c|}
\hline \multicolumn{7}{|c|}{ PSNR (PEAK SIGNAL TO NOISE RATIO) } \\
\hline NOISE & $\begin{array}{c}\text { NOISE } \\
\text { VARIANCE }\end{array}$ & $\begin{array}{c}\text { MEAN } \\
\text { FILTER }\end{array}$ & $\begin{array}{c}\text { MEDIAN } \\
\text { FILTER }\end{array}$ & $\begin{array}{l}\text { UNIVERSAL } \\
\text { THRESHOLD }\end{array}$ & $\begin{array}{c}\text { VISU } \\
\text { SHRINK }\end{array}$ & $\begin{array}{c}\text { BAYES } \\
\text { SHRINK }\end{array}$ \\
\hline \multirow{6}{*}{ 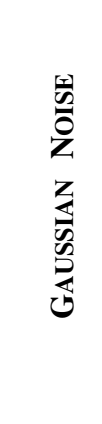 } & 0.001 & 24.0598 & 25.4934 & 27.2016 & 28.2978 & 33.7031 \\
\hline & 0.002 & 23.2251 & 24.3480 & 25.1748 & 26.1439 & 29.9001 \\
\hline & 0.003 & 22.5261 & 23.4147 & 24.0062 & 24.8430 & 27.7650 \\
\hline & 0.004 & 21.9796 & 22.6049 & 23.1590 & 23.8149 & 26.0865 \\
\hline & 0.005 & 21.4536 & 22.0205 & 22.5099 & 23.0527 & 25.1235 \\
\hline & 0.01 & 19.5569 & 19.7703 & 20.3580 & 20.5660 & 22.0446 \\
\hline \multirow{6}{*}{ 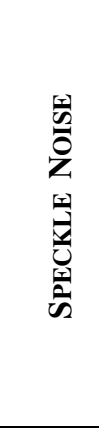 } & 0.001 & 24.8274 & 26.6157 & 28.4073 & 32.6526 & 44.0220 \\
\hline & 0.002 & 24.5114 & 26.1260 & 26.8834 & 30.4768 & 40.0535 \\
\hline & 0.003 & 24.2207 & 25.6708 & 25.9557 & 29.3585 & 38.3935 \\
\hline & 0.004 & 23.9316 & 25.2771 & 25.3274 & 28.1881 & 35.6827 \\
\hline & 0.005 & 23.7015 & 24.8599 & 24.8691 & 27.5283 & 34.3460 \\
\hline & 0.01 & 22.6357 & 23.4053 & 23.3231 & 25.1853 & 30.9207 \\
\hline
\end{tabular}



Figure4. Comparison of PSNR for cameraman image (corrupted with Gaussian noise) at different noise variance 
The International Journal of Multimedia \& Its Applications (IJMA) Vol.6, No.3, June 2014

Table2. Comparison of MSE for Cameraman image corrupted with Gaussian and Speckle noise at different Noise variances using db1

\begin{tabular}{|c|c|c|c|c|c|c|}
\hline \multicolumn{7}{|c|}{ MSE (MEAN SQUARE ERROR) } \\
\hline NOISE & $\begin{array}{c}\text { NOISE } \\
\text { VARIANCE }\end{array}$ & $\begin{array}{c}\text { MEAN } \\
\text { FILTER }\end{array}$ & $\begin{array}{c}\text { MEDIAN } \\
\text { FILTER }\end{array}$ & $\begin{array}{l}\text { UNIVERSAL } \\
\text { THRESHOLD }\end{array}$ & $\begin{array}{c}\text { VISU } \\
\text { SHRINK }\end{array}$ & $\begin{array}{c}\text { BAYES } \\
\text { SHRINK }\end{array}$ \\
\hline \multirow{6}{*}{ 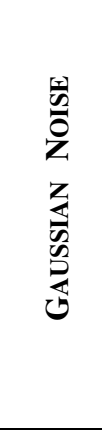 } & 0.001 & 255.3265 & 183.5446 & 123.8560 & 96.2288 & 27.7188 \\
\hline & 0.002 & 309.4321 & 238.9368 & 197.5136 & 158.0136 & 66.5377 \\
\hline & 0.003 & 363.4693 & 296.2178 & 258.5006 & 213.1975 & 108.7875 \\
\hline & 0.004 & 412.2133 & 356.9362 & 314.1828 & 270.1428 & 160.1160 \\
\hline & 0.005 & 465.2894 & 408.3482 & 364.8271 & 321.9641 & 199.8629 \\
\hline & 0.01 & 720.1005 & 685.5656 & 598.8007 & 570.7912 & 406.0842 \\
\hline \multirow{6}{*}{ 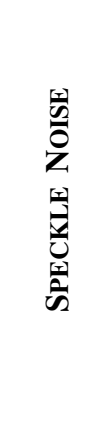 } & 0.001 & 213.9645 & 141.7451 & 93.8319 & 35.3036 & 2.5756 \\
\hline & 0.002 & 230.1138 & 158.6638 & 133.2721 & 58.2642 & 6.4229 \\
\hline & 0.003 & 246.0413 & 176.1971 & 165.0083 & 75.3748 & 9.4130 \\
\hline & 0.004 & 262.9796 & 192.9158 & 190.6971 & 98.6903 & 17.5716 \\
\hline & 0.005 & 277.2851 & 212.3693 & 211.9193 & 114.8823 & 23.9047 \\
\hline & 0.01 & 354.4109 & 296.8613 & 302.5347 & 197.0393 & 52.6035 \\
\hline
\end{tabular}

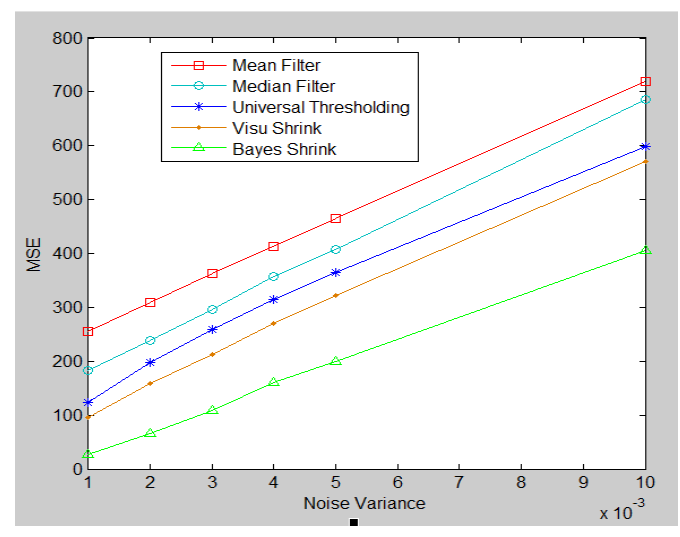

Figure5. Comparison of MSE for cameraman image (corrupted with Gaussian noise) at different noise variances 
The cameraman image is corrupted by gaussian noise of variance 0.01 and results obtained using filters and wavelets have been shown in Figure 6.

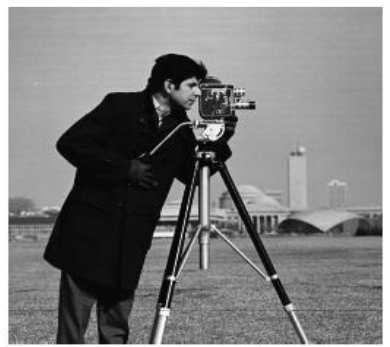

(a)

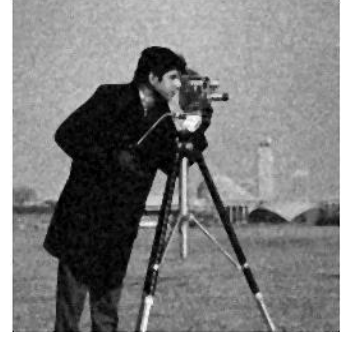

(d)

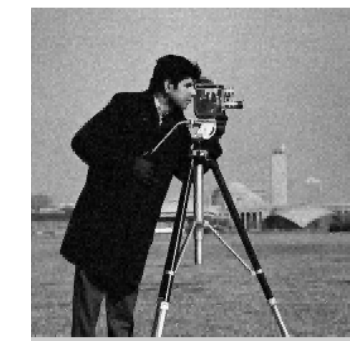

$(\mathrm{g})$

$(\mathrm{g})$

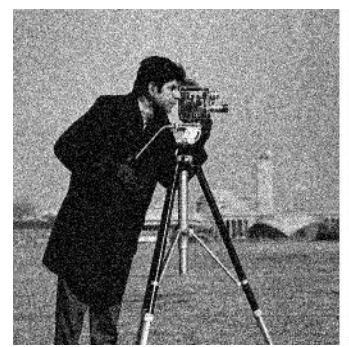

(b)

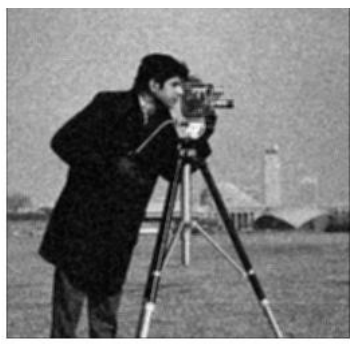

(c)



(e)

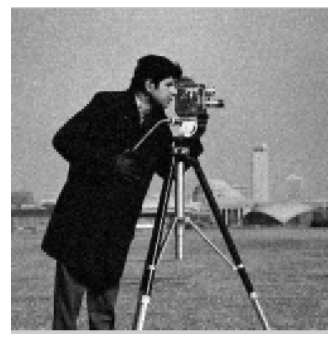

(f)

Figure 6. Denoising of cameraman image corrupted by Gaussian noise of variance 0.01

(a) Original image (b) Noisy image (c) Mean Filter (d) Median Filter (e) Universal thresholding (f) Visu Shrink (g) Bayes shrink

Table 3. shows the comparison of PSNR, MSE and Mean Absolute Error (MAE) for cameraman image at different decomposition levels. As number of levels increased, PSNR gets decreased whereas MAE and MSE get increased. Figure 7, 8 and 9 show that decomposition level1 has high PSNR and low MSE and MAE than other decomposition levels. 
The International Journal of Multimedia \& Its Applications (IJMA) Vol.6, No.3, June 2014

Table3. Comparison of PSNR, MSE and MAE for Cameraman image corrupted with Gaussian noise at different decomposition levels using $\mathrm{db} 2$

\begin{tabular}{|c|c|c|c|c|c|c|c|}
\hline \multirow{2}{*}{ 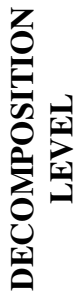 } & \multirow{2}{*}{$\begin{array}{c}\text { NOISE } \\
\text { VARIANCE }\end{array}$} & \multicolumn{3}{|c|}{$\begin{array}{l}\text { UNIVERSAL } \\
\text { THRESHOLD }\end{array}$} & \multicolumn{3}{|c|}{ VISU SHRINK } \\
\hline & & $\begin{array}{l}\text { PSNR } \\
(\text { db })\end{array}$ & MSE & MAE & $\begin{array}{l}\text { PSNR } \\
(\text { db) }\end{array}$ & MSE & MAE \\
\hline \multirow{5}{*}{ 氠 } & 0.001 & 27.417 & 117.864 & 7.9166 & 28.031 & 102.305 & 7.512 \\
\hline & 0.002 & 25.483 & 183.956 & 10.070 & 26.028 & 162.286 & 9.622 \\
\hline & 0.003 & 24.324 & 240.229 & 11.632 & 24.764 & 217.077 & 11.235 \\
\hline & 0.005 & 22.775 & 343.185 & 14.090 & 23.087 & 319.419 & 13.763 \\
\hline & 0.01 & 20.610 & 564.927 & 18.373 & 20.740 & 548.297 & 18.179 \\
\hline \multirow{5}{*}{ 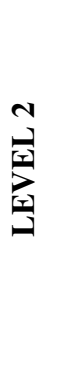 } & 0.001 & 25.736 & 173.564 & 9.612 & 26.778 & 136.524 & 8.767 \\
\hline & 0.002 & 23.834 & 268.933 & 12.177 & 24.667 & 222.007 & 11.343 \\
\hline & 0.003 & 22.673 & 351.355 & 14.047 & 23.477 & 291.968 & 13.110 \\
\hline & 0.005 & 21.144 & 499.579 & 17.007 & 21.769 & 432.649 & 16.135 \\
\hline & 0.01 & 19.027 & 813.403 & 22.095 & 19.424 & 742.403 & 21.331 \\
\hline \multirow{5}{*}{ 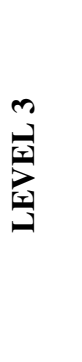 } & 0.001 & 25.201 & 196.329 & 10.250 & 26.473 & 146.467 & 9.1091 \\
\hline & 0.002 & 23.203 & 311.007 & 13.050 & 24.386 & 236.814 & 11.730 \\
\hline & 0.003 & 22.037 & 406.731 & 15.079 & 23.109 & 317.751 & 13.722 \\
\hline & 0.005 & 20.532 & 575.175 & 18.176 & 21.422 & 468.585 & 16.764 \\
\hline & 0.01 & 18.443 & 930.519 & 23.619 & 19.091 & 801.536 & 22.171 \\
\hline
\end{tabular}


The International Journal of Multimedia \& Its Applications (IJMA) Vol.6, No.3, June 2014

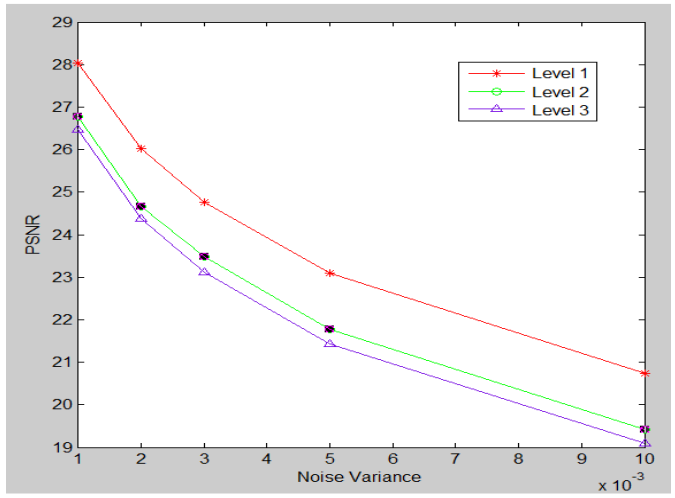

Figure 7. Comparison of Peak Signal to Noise Ratio (PSNR) for cameraman image (denoising using Visu Shrink) at different decomposition levels

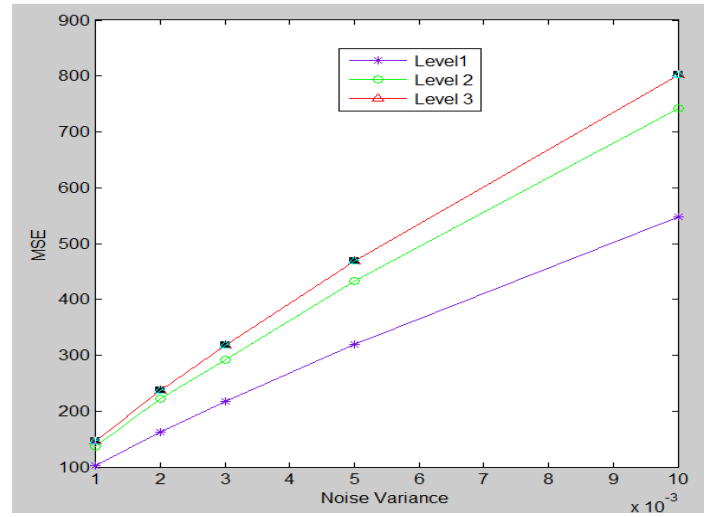

Figure 8. Comparison of Mean Square Error (MSE) for cameraman image (denoising using Visu Shrink) at different decomposition levels

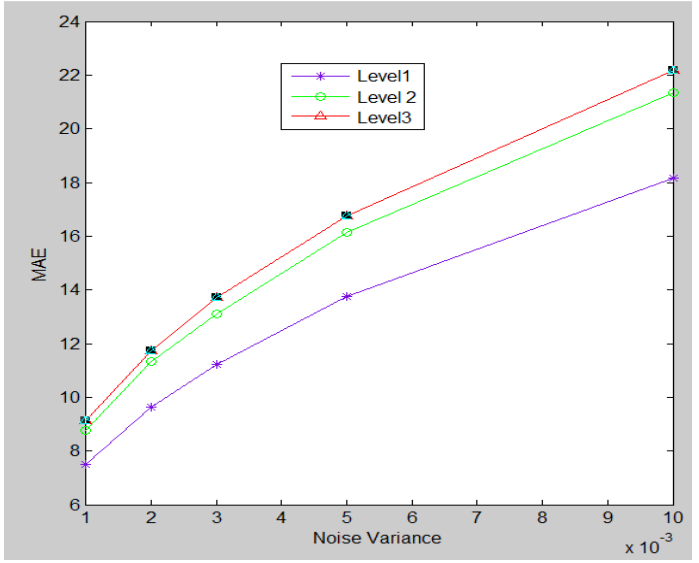

Figure 9. Comparison of Mean Absolute Error (MAE) for cameraman image (denoising using Visu Shrink) at different decomposition levels 
The International Journal of Multimedia \& Its Applications (IJMA) Vol.6, No.3, June 2014

\section{CONCLUSION}

In this paper, an analysis of denoising techniques like filters and wavelet methods has been carried out. Filtering is done by Mean and Median Filter. And three different wavelet thresholding techniques have been discussed i.e. Universal Thresholding, Bayes Shrink and Visu Shrink. The results conclude that Bayes shrinkage method has high PSNR at different noise variance and low MSE. Also the comparison of Wavelet thresholding methods at different decomposition level has been discussed. From simulation result, it is evident that decomposition level 1 has high PSNR and low MAE and MSE than other decomposition levels i.e. level 2 and level 3.This concludes that decomposition level 1 is better in removing Gaussian noise than other decomposition levels.

\section{REFERENCES}

[1] Rajni, Anutam, "Image Denoising Techniques -An Overview," International Journal of Computer Applications (0975-8887), Vol. 86, No.16, January 2014.

[2] Akhilesh Bijalwan, Aditya Goyal and Nidhi Sethi, "Wavelet Transform Based Image Denoise Using Threshold Approaches," International Journal of Engineering and Advanced Technology (IJEAT), ISSN: 2249-8958, Vol.1, Issue 5, June 2012.

[3] S.Arivazhagan, S.Deivalakshmi, K.Kannan, "Performance Analysis of Image Denoising System for different levels of Wavelet decomposition,” International Journal of Imaging Science and Engineering (IJISE), Vol.1, No.3, July 2007.

[4] Pawan Patidar, Manoj Gupta,Sumit Srivastava, Ashok Kumar Nagawat, "Image De-noising by Various Filters for Different Noise," International Journal of Computer Applications, Vol.9, No.4, November 2010.

[5] Mohammed Ghouse, Dr.M.Siddappa, "Adaptive Techniques Based High Impulsive Noise Detection And Reduction of a Digital Image,”Journal of Theoretical and Applied Information Technology.

[6] Jappreet Kaur, Manpreet Kaur, Poonamdeep Kaur, Manpreet Kaur, "Comparative Analysis of Image Denoising Techniques," International Journal of Emerging Technology and Advanced Engineering, ISSN 2250-2459, Vol. 2, Issue 6, June 2012.

[7] Govindaraj.V, Sengottaiyan.G , "Survey of Image Denoising using Different Filters," International Journal of Science, Engineering and Technology Research (IJSETR), Vol.2, Issue 2, February 2013.

[8] Idan Ram, Michael Elad, "Generalized Tree-Based Wavelet Transform," IEEE Transactions On Signal Processing, Vol. 59, No. 9, September 2011.

[9] Rakesh Kumar and B.S.Saini, "Improved Image Denoising Techniques Using Neighbouring Wavelet Coefficients of Optimal Wavelet with Adaptive Thresholding," International Journal of Computer Theory and Engineering, Vol.4, No.3, June 2012.

[10] Sethunadh R and Tessamma Thomas, "Spatially Adaptive image denoising using Undecimated Directionlet Transform," International Journal of Computer Applications, Vol.84, No. 11,December 2013

[11] S.Kother Mohideen Dr. S. Arumuga Perumal, Dr. M.Mohamed Sathik , “ Image De-noising using Discrete Wavelet transform," IJCSNS International Journal of Computer Science and Network Security, Vol.8 No.1, January 2008.

[12] Savita Gupta, R.C. Chauhan and Lakhwinder Kaur, "Image denoising using Wavelet Thresholding," ICVGIP 2002, Proceedings of the Third Indian Conference on Computer Vision, Graphics Image Processing, Ahmedabad, India, 2002

[13] S.Grace Chang, Bin Yu, Martin Vetterli , "Adaptive Wavelet Thresholding for image denoising and compression,” IEEE Transaction On Image Processing, Vol.9, No.9, September 2000

[14] Nilanjan Dey, Pradipti Nandi, Nilanjana Barman, Debolina Das, Subhabrata Chakraborty ,“ A Comparative Study between Moravec and Harris Corner Detection of Noisy Images Using Adaptive Wavelet Thresholding Technique," International Journal of Engineering Research and Applications (IJERA), ISSN: 2248-9622, Vol. 2, Issue 1, Jan-Feb 2012.

[15] Tajinder Singh, Rajeev Bedi, "A Non - Linear Approach For Image De-Noising Using Different Wavelet Thresholding," International Journal of Advanced Engineering Research and Studies, ISSN2249-8974,Vol.1,Issue3,April-June,2012 
The International Journal of Multimedia \& Its Applications (IJMA) Vol.6, No.3, June 2014

[16] Abdolhossein Fathi and Ahmad Reza Naghsh-Nilchi, "Efficient Image Denoising Method Based on a New Adaptive Wavelet Packet Thresholding Function," IEEE Transaction On Image Processing, Vol. 21, No. 9, September 2012

[17] Virendra Kumar, Dr. Ajay Kumar, "Simulative Analysis of Image denoising using Wavelet ThresholdingTechnique," International Journal of Advanced Research in Computer Engineering and Technology (IJARCET), Vol.2, No.5, May 2013

[18] Mark J.T. Smith and Steven L. Eddins, “Analysis/SynthesisTechniques for subband image coding, IEEE Trans. Acoustic Speech and Signal Processing, Vol.38, No.8, Aug 1990

[19] D.L. Donoho and I.M. Johnstone, "Denoising by soft thresholding," IEEE Trans. on Information Theory, Vo.41, 1995

[20] Raghuveer M. Rao, A.S. Bopardikar Wavelet Transforms: Introduction to Theory and Application published by Addison-Wesley, 2001

[21] S.Sutha, E. Jebamalar Leavline, D. ASR Antony Gnana Singh, " A Comprehensive Study on Wavelet based Shrinkage Methods for Denoising Natural Images," WSEAS Transactions on Signal Processing, Vol. 9, Issue 4, October 2013

[22] E.Jebamalar Leavline, S.Sutha, D.Asir Antony Gnana Singh, "Wavelet Domain Shrinkage Methods for Noise Removal in Images: A Compendium," International Journal of Computer Applications,Vol.33, No.10, November 2011

[23] G.Y. Chen, T.D. Bui, A. Krzyak, "Image denoising using neighbouring Wavelet coefficients," Acoustics Speech and Signal processing, IEEE International Conference, Vol.2, May 2004

\section{AUTHORS}

\section{Anutam}

She is currently pursuing M.Tech from SBS State TechnicalCampus, Ferozepur, India. She has completed B.Tech from PTU, Jalandhar in 2012. Her areas of interest includesWireless Communication and Image Processing.

\section{Rajni}

She is currently Associate Professor at SBS State Technical Campus Ferozepur, India. She has completed her M.E. from NITTTR, Chandigarh, India and B.Tech from REC, NIT, Kurukshetra, India. She has sixteen years of academic experience and two years industrial experience. She has authored a number of research papers in International journals, National and International conferences. Her areas of interest include wireless communication, signal Processing and Antenna design.
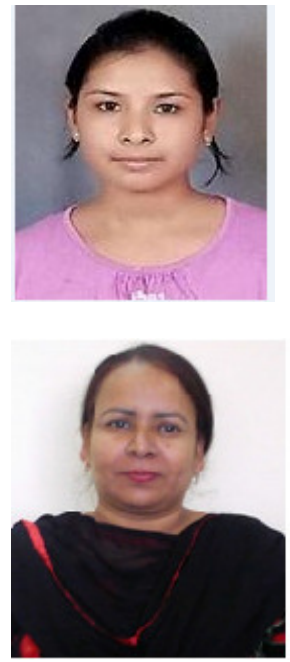